Journal of Environmental Sciences (JES)

Institute of Environmental Studies and Research, Ain Shams University

Mohamed, Asmaa et al.

\title{
EFFECT OF FOLIAR SPRAY (ALGAE EXTRACT), COMPOST AND BIO FERTILIZER ON WHEAT PRODUCTIVITY
}

\author{
Asmaa,A. Mohamed ${ }^{(1)}$; Ezzat M. Soliman ${ }^{(2)}$ \\ and Usama S. El-Bialy (1)
}

1) Soil, Water and Environment Research Institute, Agric. Res. Center, Giza, Egypt 2) Institute of Studies and Environmental Researches, Ain Shams Univ. Cairo, Egypt.

\begin{abstract}
A field experiment in the form of split-split plot design with three replications was conducted at Mashtool ElSouk, Al- Sharkia Governorate, Egypt during 2016/2017 and 2017/2018 growing seasons. The aim of study is to determine the effect of different foliar spray (Algae extract) level (without, 0.50 and $0.75 \mathrm{~cm} / \mathrm{l}$ ) and different compost levels (without, 6 and 8 ton/fad) in absence or presence of biofertilizer on wheat yield

The results can be summarized as follows:

1- Data indicate that there are significant differences of foliar concentrations as algae extract, compost levels and bio fertilizer for all studied characters in the two seasons.

2- Spraying wheat plants with algae extract at the highest concentration $(0.75$ $\mathrm{cm} / \mathrm{L}$ ) gave a significant increase of most yield components as well as grain straw and biological yields compared to the other two concentrations with exception of grain no./spike which no addition of algae extract recorded the highest significant values of all characteristics in both seasons

3 -The addition of compost at the highest rate ( 8 ton/fad) resulted in a significant increase of most yield components and wheat yields comparing with the other two rates except the parameter of grain no./spike as mentioned before. Such results were the same in two seasons.

4-The addition of biofertilizer caused the highest significant values of the
\end{abstract}


above mentioned characteristics except for grain no./spike which the highest values of such character resulted from no addition of biofertilizer. The two seasons had the same results.

5-It must be mentioned that the interaction effect among the three factors under study took the same trend of such individual factors where the application of algae extract at the level of $0.75 \mathrm{~cm} / \mathrm{L}$ and compost level 8 ton /fad as well as applied biofertilizer resulted in the highest significant values of all parameters mentioned before, with exception of grain no./spike which was the highest with no addition of any factors under study, such results were similar in both seasons.

\section{INTRODUCTION}

Wheat (Triticum aestivum L.) is one of the most important food grain crops grown in the world, which has been used as food since prehistoric times. Wheat is considered a staple food for over 10 billion people in as many as 43 countries of the world and it provides about $20 \%$ of the total food calories for human race (Reddy, 2004). In Egypt, wheat is cultivated on an area of 1.343 million hectares seasonally with an annual production of 8.800 million ton, with average yield of $6.55 \mathrm{t} / \mathrm{ha}$ (FAO, 2020).

All over the world, intensive cultivation methods were found to remove higher quantities of elements from the soil reservoir. Both of macro and micronutrients are essential for developing plants and improving its yield characteristics, since it serves as co-enzymes and in the redox systems for essential processes in the plant cell performance (Hall and Williams, 2003 and Imran and Gurmani, 2012). 
Journal of Environmental Sciences (JES)

Institute of Environmental Studies and Research, Ain Shams University

Mohamed, Asmaa et al.

Nowadays scientific studies have mentioned threat of using chemical fertilizers and its harmful effects on the human and environmental health. Therefore, relying on the concept of sustainable agriculture was necessary to replace the use of chemical fertilizers with organic and bio- fertilizers, which can improve physiological performance and productivity of plants, in addition it's a low cost, renewable and environmentally friendly source. The information about agricultural use of algae extract as a bio-fertilizer in wheat plants are little. With reference to the chemical analysis of algae extract, Zhang and Ervin (2004) revealed that a wide diversity of plant growth regulators such as cytokinins and auxins, in addition to the presence of micro elements i.e $\mathrm{Fe}, \mathrm{Mn}, \mathrm{Zn}$ and $\mathrm{Cu}$ were used. Earlier researchers showed that using algae as foliar or soil application enhanced physiological performance, where it contains of essential growth hormones and nutrients, which are important to increase productivity (Prasad et al., 2010 and Latique et al., 2013). In previous studies, many researchers recommended algae extract as foliar application for enhancing the growth parameters of potato (Awad et al., 2006), mung bean (Pramanick et al., 2013) and garlic plants (Shalaby and ElRamady, 2014). More, Karthikeyan and Shanmugam (2015) showed that spraying algae as foliar bio-fertilizer recorded a relative increase $51 \%$ in Peanut and sunflower seed yield. Concerning the use of most superior algae concentration as foliar application, Furthermore, Shaaban et al. (2010) in his study on the effect of fertilization with algae extract on wheat nutrient balance, showed that application of modified algae extract at $2 \mathrm{gm} / 1$ resulted 
in the superiority of $\mathrm{N}, \mathrm{P}, \mathrm{K}$ and Mg-uptakes. While, Nofal et al. (2016) reported that using Alga at $300 \mathrm{~g} / \mathrm{fed}$ as foliar application significantly increased the Zinc, Iron, Copper, Manganese content, and it also improved protein and nitrogen contents of maize grains. Mansour et al. (2019) cleared that the most effective dose of using algae on wheat plant was $1.5 \mathrm{~g} / \mathrm{L}$ which led to increasing grains number/spike, spikes number/m2, 1000- grain weight, straw yield/fed and grain yield/fed.

Biofertilizer is frequently recommended firstly for improving biological, physical and chemical properties of soil and secondly to get clean agricultural products free of undesirable doses of heavy metals and other pollutants (ELHabbasha et al.,2007). Rock P and K materials either applied singly or in combination did not significantly enhance soil availability of $\mathrm{P}$ and $\mathrm{K}$, but inoculation of phosphorus and potassium with PSB (phosphorus solving bacteria) has increased higher soil $\mathrm{P}$ availability than KSB (potassium solving bacteria) which was recommended as a K- solubilizer. Inoculation of these bacteria in conjunction with amendment of its respective rock $\mathrm{P}$ or $\mathrm{K}$ materials increased the availability of $\mathrm{P}$ and $\mathrm{K}$ in soil.

The aim of this study is to assess the effect of Algae extract as foliar application and to investigate the effect of compost and bio fertilizer as soil application on wheat productivity. 


\section{MATERIALS AND METHODS}

A field experiment was performed in Al-Betiah village, Mashtoul ElSouk District, Sharqiah Governorate during two successive winter seasons 2016/2017 and 2017/2018 to study the effect of different foliar spray (Algae extract) and different compost levels in absence or presence of biofertilizer on wheat yield and yield components.. Grains of wheat (variety sids 12), were sown at the rate of $70 \mathrm{~kg} / \mathrm{fad}$ in November $20^{\text {th }}$ and $25^{\text {th }}$ in the first and second season, respectively. The area of each plot was $10.5 \mathrm{~m}^{2}$ (1/400 fed.)

The experimental plots were statistically arranged in split, split plot design with three replicates. The treatments were as follows:

\section{1- The treatments occupied the main plots were:}

*Spraying Algae extracts (zero rate).

* Spraying Algae extracts $(0.50 \mathrm{~cm} / \mathrm{l})$

* Spraying Algae extracts $(0.75 \mathrm{~cm} / \mathrm{l})$

Wheat plants were sprayed with algae extract twice at 45 and 60 days age. The plants of Fadden were sprayed with $200 \mathrm{~L}$

\section{2- The treatments occupied the sub-main plots were:}

*compost (zero rate)

*compost 1 (6 ton/fed)

* compost 2 (8 ton/fed) 
Table 1.: Some physical and chemical analysis of compost.

\begin{tabular}{|l|c|c|}
\hline Properties & Units & Value \\
\hline Weight of cubic meter & $\mathrm{Kg}$ & 565 \\
\hline Moisture content \% & $\%$ & 35 \\
\hline PH $1: 10$ & & 7.95 \\
\hline EC $1: 10$ & $\mathrm{dsm}^{-1}$ & 3.85 \\
\hline Ammonium (NH4) & $\mathrm{mg} \mathrm{kg}^{-1}$ & 695 \\
\hline Nitrate (NO3) & 0 & 0 \\
\hline Total nitrogen & $\%$ & 1.00 \\
\hline Organic matter & $\%$ & 33.00 \\
\hline Organic carbon & $\%$ & 18.65 \\
\hline Ash & $\%$ & 67.85 \\
\hline C $:$ N ratio & & $1: 18.85$ \\
\hline Total phosphorus & $\%$ & 0.80 \\
\hline Total potassium & $\%$ & 1.05 \\
\hline All analys ar caula & \\
\hline
\end{tabular}

All analysis are calculated on dry weight basic, except weight and moisture content.

\section{3- The treatments occupied the sub-sub. Main plots were:}

*without biofertilizer

\section{*Biofertilizer}

Biological fertilizers: The used biological fertilizers were cerialine as nitrogen `fixer and Bacills megaterium as phosphate dissolving bacteria and Bacillus circulans as potassium dissolving bacteria. Bio fertilizers were added mixed with sand and broadcasted on the soil of specific plots according to the recommending source of such biofertilizers from the ARC, Ministry of Agriculture, Cairo Egypt. 
Sampling technique: At harvest, a sample of $1 \mathrm{~m}^{2}$ intermediate row was chosen at random from each treatment in three replicates and were harvested to determine the following yield component parameters :

Plant height (cm), Wt. of grain/spike (g), Grain no./spike, spike no./m², Wt. of 1000 grains $(\mathrm{g})$ and Harvest index \%

Yield: Grain, Straw and Biological yields (kg/fed) were determined.

Soil sampling: Soil samples of each site at a depth of 0-30 cm were collected before application of treatments in each season and analyzed to determine some soil physical and chemical properties which were in Table (2). All samples were air dried, grounded, screened through $2 \mathrm{~mm}$ sieve and analyzed. 
Table (2): Some physical and chemical characteristics of the experimental soil of both seasons.

\begin{tabular}{|c|c|c|c|}
\hline \multirow{2}{*}{\multicolumn{2}{|c|}{ Mechanical analysis }} & \multicolumn{2}{|c|}{ Winter season } \\
\hline & & 2016/2017 & $2017 / 2018$ \\
\hline Sand & \multirow{3}{*}{$\%$} & 21.60 & 21 \\
\hline Silt & & 39.50 & 39.80 \\
\hline Clay & & 38.90 & 39.20 \\
\hline \multicolumn{2}{|c|}{ Soil texture } & Clay loam & Clay loam \\
\hline \multicolumn{4}{|c|}{$\begin{array}{l}\text { Chemical analysis } \\
\end{array}$} \\
\hline \multicolumn{2}{|c|}{ pH soil water susp., $1: 2.5$} & 7.73 & 7.76 \\
\hline \multicolumn{2}{|c|}{ EC, dsm-1 soil : water extr., $1: 5$} & 1.95 & 2.35 \\
\hline S.P & \multirow[t]{3}{*}{ (1) } & 59 & 60 \\
\hline $\mathrm{CaCO}_{3}$ & & 4.60 & 5.30 \\
\hline $\mathrm{OM}$ & & 0.51 & 0.55 \\
\hline $\mathrm{Ca}^{++}$ & \multirow{8}{*}{$\mathrm{Meq}^{-1}$} & 4.00 & 3.03 \\
\hline $\mathrm{Mg}^{++}$ & & 2.50 & 2.00 \\
\hline $\mathrm{K}^{+}$ & & 0.30 & 0.80 \\
\hline $\mathrm{Na}^{+}$ & & 12.20 & 17.10 \\
\hline $\mathrm{CO}_{3}^{--}$ & & 0.0 & 0.0 \\
\hline $\mathrm{HCO}_{3}^{-}$ & & 0.4 & 0.7 \\
\hline $\mathrm{Cl}^{-}$ & & 14.10 & 20.00 \\
\hline $\mathrm{SO}_{4}^{--}$ & & 3.55 & 2.35 \\
\hline $\mathrm{N}$ & \multirow{3}{*}{ Available $\left(\mathrm{mg} \mathrm{kg}^{-1}\right)$} & 40.60 & 43.55 \\
\hline $\mathrm{P}$ & & 5.55 & 6.00 \\
\hline $\mathrm{K}$ & & 330 & 355 \\
\hline
\end{tabular}


Journal of Environmental Sciences (JES)

Institute of Environmental Studies and Research, Ain Shams University

Mohamed, Asmaa et al.

\section{Methods of Soil analysis}

A. Soil texture, $\mathrm{pH}$ and Ec values were determined according to Ryan et al., (1996) as follows:

1-Soil texture was determined by pipette method.

2-pH values were measured using $\mathrm{pH}$ meter in soil suspension 1:2.5

3-Ec values were measured using Ec meter in soil suspension 1:5

B- Calcium carbonate $(\mathrm{CaCo} 3)$, organic matter and water soluble ions were determined according to Ryan et al., (1996).

C- Available nutrients in soil:

1-Available $\mathrm{N}$ was determined using Kjeldahl method (A.O.A.C, 1970).

2-Available phosphorus was determined using Olsen method (Jackson, 1973).

3-Available potassium was determined by flame-photo-metrically (Black, 1982).

Statistical analysis: The results were statistically analyzed using Mstat computer package to calculate $\mathrm{F}$ ratio according to Snedecor and Cochran (1982). Least significant differences method (L.S.D) was used to differentiate means at the 0.05 level (Waller and Duncan, 1969)

\section{RESULTS AND DISCUSSION}

\section{Yield components}

Data presented in Tables $(3,4,5,6,7)$ showed that all wheat yield components i.e. plant height $(\mathrm{cm})$, wt. of grains/spike $(\mathrm{g})$, grain no. /spike, spike no./m2 and wt.of 1000 grain(g). Significantly affected by all factors 
under study i.e. foliar algae extract and application of compost and biofertilizer to soil.

Table (3): Mean values of plant height $(\mathrm{cm})$ as affected by spray, compost and bio in 2016/2017 and 2017/2018 growing seasons.

\begin{tabular}{|c|c|c|c|c|c|c|c|}
\hline \multirow{3}{*}{ Spray } & \multirow{3}{*}{ Compost } & \multicolumn{3}{|c|}{ Season $2016 / 2017$} & \multicolumn{3}{|c|}{ Season $2017 / 2018$} \\
\hline & & \multicolumn{6}{|c|}{ Mean height } \\
\hline & & $\begin{array}{c}\text { Without } \\
\text { Bio }\end{array}$ & $\begin{array}{l}\text { With } \\
\text { Bio }\end{array}$ & $\begin{array}{l}\text { Total } \\
\text { Mean }\end{array}$ & $\begin{array}{c}\text { Without } \\
\text { Bio }\end{array}$ & $\begin{array}{l}\text { with } \\
\text { Bio }\end{array}$ & $\begin{array}{l}\text { Total } \\
\text { Mean }\end{array}$ \\
\hline \multirow{3}{*}{ Without } & Without & 73.00 & 76.00 & 74.50 & 78.00 & 80.33 & 79.17 \\
\hline & 6ton/fad & 79.33 & 83.00 & 81.17 & 83.33 & 87.00 & 85.17 \\
\hline & 8ton/fad & 81.67 & 85.33 & 83.50 & 85.67 & 89.67 & 87.67 \\
\hline \multicolumn{2}{|c|}{ Total Mean } & 78.00 & 81.44 & 79.72 & 82.33 & 85.67 & 84.00 \\
\hline \multirow{3}{*}{$0.50 \mathrm{~cm} / \mathrm{l}$} & Without & 77.00 & 86.00 & 81.50 & 81.33 & 90.33 & 85.83 \\
\hline & 6ton/fad & 88.00 & 90.67 & 89.33 & 92.00 & 94.67 & 93.33 \\
\hline & 8ton/fad & 91.33 & 95.33 & 93.33 & 95.33 & 99.33 & $\mathbf{9 7 . 3 3}$ \\
\hline \multicolumn{2}{|c|}{ Total Mean } & 85.44 & 90.67 & 88.06 & 89.56 & 94.78 & 92.17 \\
\hline \multirow{3}{*}{$0.75 \mathrm{~cm} / \mathrm{l}$} & Without & 78.00 & 87.00 & 82.50 & 82.33 & 91.00 & 86.67 \\
\hline & 6ton/fad & 89.67 & 93.00 & 91.33 & 93.67 & 97.00 & 95.33 \\
\hline & 8ton/fad & 92.33 & $\begin{array}{c}100.0 \\
0\end{array}$ & 96.17 & 96.33 & $\begin{array}{c}104.0 \\
0\end{array}$ & 100.17 \\
\hline \multicolumn{2}{|c|}{ Total Mean } & 86.67 & 93.33 & 90.00 & 90.78 & $\mathbf{9 7 . 3 3}$ & 94.06 \\
\hline \multicolumn{2}{|c|}{ Grand mean } & 83.37 & 88.48 & 85.93 & 87.56 & 92.59 & 90.07 \\
\hline \multirow{7}{*}{ LSD $_{0.05}$} & $\begin{array}{l}\text { Spray }(S) \\
=\end{array}$ & \multicolumn{3}{|l|}{1.532} & \multicolumn{3}{|l|}{1.27} \\
\hline & $\begin{array}{l}\text { Compos } \\
\mathrm{t}(\mathrm{C}=\end{array}$ & \multicolumn{3}{|l|}{1.489} & \multicolumn{3}{|l|}{1.21} \\
\hline & Bio $(B)=$ & \multicolumn{3}{|l|}{$* *$} & \multicolumn{3}{|l|}{$* *$} \\
\hline & $\mathbf{S} \times \mathbf{C}=$ & \multicolumn{3}{|l|}{ Ns } & \multicolumn{3}{|l|}{2.09} \\
\hline & $\mathbf{S} \times \mathbf{B}=$ & \multicolumn{3}{|l|}{1.577} & \multicolumn{3}{|l|}{1.34} \\
\hline & $\mathrm{C} \times \mathrm{B}=$ & \multicolumn{3}{|l|}{1.577} & \multicolumn{3}{|l|}{1.34} \\
\hline & $\begin{array}{l}\mathbf{S} \times \mathbf{C} \times \mathbf{B} \\
=\end{array}$ & \multicolumn{3}{|l|}{ Ns } & \multicolumn{3}{|l|}{2.32} \\
\hline
\end{tabular}


Table (4): Mean values of Wt of grains/spike ( $\mathrm{g}$ ) as affected by spray, compost and bio in 2016/2017 and 2017/2018 growing seasons.

\begin{tabular}{|c|c|c|c|c|c|c|c|}
\hline \multirow{3}{*}{ Spray } & \multirow{3}{*}{ Compost } & \multicolumn{3}{|c|}{ Season $2016 / 2017$} & \multicolumn{3}{|c|}{ Season 2017/2018 } \\
\hline & & \multicolumn{6}{|c|}{ Mean Wt of grains/spike } \\
\hline & & $\begin{array}{l}\text { Witho } \\
\text { ut Bio }\end{array}$ & $\begin{array}{l}\text { With } \\
\text { Bio } \\
\end{array}$ & $\begin{array}{l}\text { Total } \\
\text { Mean }\end{array}$ & $\begin{array}{l}\text { Witho } \\
\text { ut Bio }\end{array}$ & $\begin{array}{l}\text { With } \\
\text { Bio } \\
\end{array}$ & $\begin{array}{l}\text { Total } \\
\text { Mean }\end{array}$ \\
\hline \multirow{3}{*}{ Without } & Without & 1.99 & 2.01 & 2.00 & 2.29 & 2.31 & 2.30 \\
\hline & 6ton/fad & 2.04 & 2.06 & 2.05 & 2.34 & 2.35 & 2.35 \\
\hline & 8ton/fad & 2.05 & 2.08 & 2.07 & 2.35 & 2.38 & 2.37 \\
\hline \multicolumn{2}{|c|}{ Total Mean } & 2.03 & 2.05 & 2.04 & 2.33 & 2.35 & 2.34 \\
\hline \multirow{3}{*}{$0.50 \mathrm{~cm} / \mathrm{l}$} & Without & 2.03 & 2.10 & 2.07 & 2.33 & 2.40 & 2.37 \\
\hline & 6ton/fad & 2.12 & 2.16 & 2.14 & 2.42 & 2.46 & 2.44 \\
\hline & 8ton/fad & 2.18 & 2.39 & 2.28 & 2.48 & 2.68 & 2.58 \\
\hline \multicolumn{2}{|c|}{ Total Mean } & 2.11 & 2.22 & 2.16 & 2.41 & 2.51 & 2.46 \\
\hline \multirow{3}{*}{$0.75 \mathrm{~cm} / \mathrm{l}$} & Without & 2.04 & 2.11 & 2.07 & 2.34 & 2.41 & 2.37 \\
\hline & 6ton/fad & 2.14 & 2.26 & 2.20 & 2.44 & 2.55 & 2.50 \\
\hline & 8ton/fad & 2.22 & 2.54 & 2.38 & 2.51 & 2.83 & 2.67 \\
\hline \multicolumn{2}{|c|}{ Total Mean } & 2.13 & 2.30 & 2.22 & 2.43 & 2.60 & 2.51 \\
\hline \multicolumn{2}{|c|}{ Grand mean } & 2.09 & 2.19 & 2.14 & 2.39 & 2.49 & 2.44 \\
\hline \multirow{7}{*}{ LSD $_{0.05}$} & $\begin{array}{l}\text { Spray (S) } \\
=\end{array}$ & \multicolumn{3}{|l|}{0.033} & \multicolumn{3}{|l|}{0.033} \\
\hline & $\begin{array}{l}\text { Compost } \\
(\mathrm{C}=\end{array}$ & \multicolumn{3}{|l|}{0.019} & \multicolumn{3}{|l|}{0.020} \\
\hline & Bio $(B)=$ & \multicolumn{3}{|l|}{$* *$} & \multicolumn{3}{|l|}{$* *$} \\
\hline & $\mathbf{S} \times \mathbf{C}=$ & \multicolumn{3}{|l|}{0.034} & \multicolumn{3}{|l|}{0.035} \\
\hline & $\mathbf{S} \times \mathbf{B}=$ & \multicolumn{3}{|l|}{0.020} & \multicolumn{3}{|l|}{0.021} \\
\hline & $\mathbf{C} \times \mathbf{B}=$ & \multicolumn{3}{|l|}{0.020} & \multicolumn{3}{|l|}{0.021} \\
\hline & $\begin{array}{l}\mathrm{S} \times \mathrm{C} \times \mathrm{B} \\
=\end{array}$ & \multicolumn{3}{|l|}{0.035} & \multicolumn{3}{|l|}{0.037} \\
\hline
\end{tabular}


Table (5): Mean values of grains no./spike as affected by spray, compost and bio in 2016/2017 and 2017/2018 growing seasons.

\begin{tabular}{|c|c|c|c|c|c|c|c|}
\hline \multirow{3}{*}{ Spray } & \multirow{3}{*}{ Compost } & \multicolumn{3}{|c|}{ Season $2016 / 2017$} & \multicolumn{3}{|c|}{ Season $2017 / 2018$} \\
\hline & & \multicolumn{6}{|c|}{ Mean grains no./spike } \\
\hline & & $\begin{array}{l}\text { Without } \\
\text { Bio }\end{array}$ & $\begin{array}{l}\text { With } \\
\text { Bio } \\
\end{array}$ & $\begin{array}{l}\text { Total } \\
\text { Mean }\end{array}$ & $\begin{array}{l}\text { Without } \\
\text { Bio }\end{array}$ & $\begin{array}{l}\text { With } \\
\text { Bio } \\
\end{array}$ & $\begin{array}{l}\text { Total } \\
\text { Mean }\end{array}$ \\
\hline \multirow{3}{*}{$\begin{array}{l}\text { With } \\
\text { out }\end{array}$} & Without & 69.00 & 66.67 & 67.83 & 73.00 & 70.67 & 71.83 \\
\hline & 6ton/fad & 65.00 & 64.00 & 64.50 & 69.00 & 68.00 & 68.50 \\
\hline & 8ton/fad & 64.33 & 63.33 & 63.83 & 68.33 & 67.33 & 67.83 \\
\hline \multicolumn{2}{|c|}{ Total Mean } & 66.11 & 64.67 & 65.39 & 70.11 & 68.67 & 69.39 \\
\hline \multirow{3}{*}{$\begin{array}{l}0.50 \mathrm{c} \\
\mathrm{m} / \mathrm{l}\end{array}$} & Without & 66.00 & 62.67 & 64.33 & 70.00 & 66.67 & 68.33 \\
\hline & 6ton/fad & 59.33 & 57.67 & 58.50 & 63.33 & 61.67 & 62.50 \\
\hline & 8ton/fad & 56.67 & 54.67 & 55.67 & 60.67 & 58.67 & 59.67 \\
\hline \multicolumn{2}{|c|}{ Total Mean } & 60.67 & 58.33 & 59.50 & 64.67 & 62.33 & 63.50 \\
\hline \multirow{3}{*}{$\begin{array}{l}0.75 \mathrm{c} \\
\mathrm{m} / \mathrm{l}\end{array}$} & Without & 65.33 & 60.67 & 63.00 & 69.33 & 64.67 & 67.00 \\
\hline & 6ton/fad & 58.33 & 57.00 & 57.67 & 62.33 & 61.00 & 61.67 \\
\hline & 8ton/fad & 57.00 & 53.00 & 55.00 & 61.00 & 57.00 & 59.00 \\
\hline \multicolumn{2}{|c|}{ Total Mean } & 60.22 & 56.89 & 58.56 & 64.22 & 60.89 & 62.56 \\
\hline \multicolumn{2}{|c|}{ Grand mean } & 62.33 & 59.96 & 61.15 & 66.33 & 63.96 & 65.15 \\
\hline \multirow{7}{*}{$\begin{array}{l}\text { LSD } \\
0.05\end{array}$} & Spray $(S)=$ & \multicolumn{3}{|l|}{0.591} & \multicolumn{3}{|l|}{0.59} \\
\hline & $\begin{array}{l}\text { Compost }(\mathrm{C} \\
=\end{array}$ & \multicolumn{3}{|l|}{0.489} & \multicolumn{3}{|l|}{0.49} \\
\hline & Bio $(\mathbf{B})=$ & \multicolumn{3}{|l|}{$* *$} & \multicolumn{3}{|l|}{$* *$} \\
\hline & $\mathrm{S} \times \mathrm{C}=$ & \multicolumn{3}{|l|}{0.849} & \multicolumn{3}{|l|}{0.85} \\
\hline & $\mathbf{S} \times \mathbf{B}=$ & \multicolumn{3}{|l|}{0.687} & \multicolumn{3}{|l|}{0.69} \\
\hline & $\mathrm{C} \times \mathrm{B}=$ & \multicolumn{3}{|l|}{0.687} & \multicolumn{3}{|l|}{0.69} \\
\hline & $\mathbf{S} \times \mathbf{C} \times \mathbf{B}=$ & \multicolumn{3}{|l|}{ Ns } & \multicolumn{3}{|l|}{ NS } \\
\hline
\end{tabular}


Table (6): Mean values of spike no. $/ \mathrm{m}^{2}$ as affected by spray, compost and bio in 2016/2017 and 2017/2018 growing seasons

\begin{tabular}{|c|c|c|c|c|c|c|c|}
\hline \multirow{3}{*}{ Spray } & \multirow{3}{*}{ Compost } & \multicolumn{3}{|c|}{ Season $2016 / 2017$} & \multicolumn{3}{|c|}{ Season $2017 / 2018$} \\
\hline & & \multicolumn{6}{|c|}{ Mean spike no./m² } \\
\hline & & $\begin{array}{l}\text { Witho } \\
\text { ut Bio }\end{array}$ & $\begin{array}{l}\text { With } \\
\text { Bio }\end{array}$ & $\begin{array}{l}\text { Total } \\
\text { Mean } \\
\end{array}$ & $\begin{array}{l}\text { Without } \\
\text { Bio }\end{array}$ & $\begin{array}{l}\text { With } \\
\text { Bio }\end{array}$ & $\begin{array}{l}\text { Total } \\
\text { Mean } \\
\end{array}$ \\
\hline \multirow{3}{*}{ Without } & Without & 260.00 & 274.33 & 267.17 & 300.00 & 313.33 & 306.67 \\
\hline & 6ton/fad & 299.33 & 318.33 & 308.83 & 333.33 & 348.00 & 340.67 \\
\hline & 8ton/fad & 307.67 & 320.67 & 314.17 & 342.67 & 356.00 & 349.33 \\
\hline \multicolumn{2}{|c|}{ Total Mean } & 289.00 & 304.44 & 296.72 & 325.33 & 339.11 & 332.22 \\
\hline \multirow{3}{*}{$0.50 \mathrm{~cm} / \mathrm{l}$} & Without & 289.33 & 328.33 & 308.83 & 324.00 & 360.00 & 342.00 \\
\hline & 6ton/fad & 338.33 & 357.67 & 348.00 & 370.67 & 386.67 & 378.67 \\
\hline & 8ton/fad & 370.67 & 404.33 & 387.50 & 400.00 & 432.00 & 416.00 \\
\hline \multicolumn{2}{|c|}{ Total Mean } & 332.78 & 363.44 & 348.11 & 364.89 & 392.89 & 378.89 \\
\hline \multirow{3}{*}{$0.75 \mathrm{~cm} / \mathrm{l}$} & Without & 295.33 & 334.00 & 314.67 & 329.33 & 366.67 & 348.00 \\
\hline & 6ton/fad & 352.00 & 395.00 & 373.50 & 381.33 & 424.00 & 402.67 \\
\hline & 8ton/fad & 378.33 & 418.33 & 398.33 & 408.00 & 449.33 & 428.67 \\
\hline \multicolumn{2}{|c|}{ Total Mean } & 341.89 & 382.44 & 362.17 & 372.89 & 413.33 & 393.11 \\
\hline \multicolumn{2}{|c|}{ Grand mean } & 321.22 & 350.11 & 335.67 & 354.37 & 381.78 & 368.07 \\
\hline \multirow{7}{*}{ LSD $_{0.05}$} & Spray $(S)=$ & \multicolumn{3}{|l|}{4.376} & \multicolumn{3}{|l|}{2.02} \\
\hline & $\begin{array}{l}\text { Compost } \\
(\mathrm{C}=\end{array}$ & \multicolumn{3}{|l|}{7.824} & \multicolumn{3}{|l|}{6.20} \\
\hline & $\operatorname{Bio}(B)=$ & \multicolumn{3}{|l|}{$* *$} & \multicolumn{3}{|l|}{$* *$} \\
\hline & $\mathbf{S} \times \mathbf{C}=$ & \multicolumn{3}{|l|}{13.552} & \multicolumn{3}{|l|}{10.74} \\
\hline & $\mathbf{S} \times \mathbf{B}=$ & \multicolumn{3}{|l|}{7.478} & \multicolumn{3}{|l|}{4.85} \\
\hline & $\mathbf{C} \times \mathbf{B}=$ & \multicolumn{3}{|l|}{ Ns } & \multicolumn{3}{|l|}{4.85} \\
\hline & $\mathbf{S} \times \mathbf{C} \times \mathbf{B}=$ & \multicolumn{3}{|l|}{ Ns } & \multicolumn{3}{|l|}{8.40} \\
\hline
\end{tabular}


Table (7): Mean values of wt.of 1000 grain (g) as affected by spray, compost and bio in 2016/2017 and 2017/2018 growing seasons

\begin{tabular}{|c|c|c|c|c|c|c|c|}
\hline \multirow{3}{*}{ Spray } & \multirow{3}{*}{ Compost } & \multicolumn{3}{|c|}{ Season $2016 / 2017$} & \multicolumn{3}{|c|}{ Season 2017/2018 } \\
\hline & & \multicolumn{6}{|c|}{ Mean wt.of 1000 grain } \\
\hline & & $\begin{array}{l}\text { With } \\
\text { out } \\
\text { Bio } \\
\end{array}$ & $\begin{array}{l}\text { With } \\
\text { Bio } \\
\end{array}$ & $\begin{array}{l}\text { Total } \\
\text { Mean }\end{array}$ & $\begin{array}{l}\text { Without } \\
\text { Bio }\end{array}$ & $\begin{array}{l}\text { With } \\
\text { Bio } \\
\end{array}$ & $\begin{array}{l}\text { Total } \\
\text { Mean }\end{array}$ \\
\hline \multirow{3}{*}{$\begin{array}{l}\text { Witho } \\
\text { ut }\end{array}$} & Without & 28.84 & 30.24 & 29.54 & 31.36 & 32.77 & 32.06 \\
\hline & 6ton/fad & 31.46 & 32.17 & 31.81 & 33.98 & 34.63 & 34.31 \\
\hline & 8ton/fad & 31.90 & 32.88 & 32.39 & 34.42 & 35.37 & 34.90 \\
\hline \multicolumn{2}{|c|}{ Total Mean } & 30.73 & 31.76 & 31.25 & 33.25 & 34.26 & 33.75 \\
\hline \multirow{3}{*}{$\begin{array}{l}0.50 \mathrm{~cm} \\
/ 1\end{array}$} & Without & 30.79 & 33.52 & 32.15 & 33.31 & 36.01 & 34.66 \\
\hline & 6ton/fad & 35.75 & 37.49 & 36.62 & 38.14 & 39.92 & 39.03 \\
\hline & 8ton/fad & 38.44 & 43.76 & 41.10 & 40.81 & 45.78 & 43.30 \\
\hline \multicolumn{2}{|c|}{ Total Mean } & 34.99 & 38.26 & 36.62 & 37.42 & 40.57 & 38.99 \\
\hline \multirow{3}{*}{$\begin{array}{l}0.75 \mathrm{~cm} \\
/ 1\end{array}$} & Without & 31.21 & 34.80 & 33.00 & 33.73 & 37.57 & 35.65 \\
\hline & 6ton/fad & 36.77 & 39.61 & 38.19 & 39.22 & 41.82 & 40.52 \\
\hline & 8ton/fad & 38.96 & 47.88 & 43.42 & 41.21 & 49.67 & 45.44 \\
\hline \multicolumn{2}{|c|}{ Total Mean } & 35.65 & 40.76 & 38.20 & 38.05 & 43.02 & 40.54 \\
\hline \multicolumn{2}{|c|}{ Grand mean } & 33.79 & 36.93 & 35.36 & 36.24 & 39.28 & 37.76 \\
\hline \multirow{7}{*}{$\begin{array}{l}\text { LSD } \\
0.05\end{array}$} & Spray $(\mathbf{S})=$ & \multicolumn{3}{|l|}{0.638} & \multicolumn{3}{|l|}{0.64} \\
\hline & $\begin{array}{l}\text { Compost }(\mathrm{C} \\
=\end{array}$ & \multicolumn{3}{|l|}{0.350} & \multicolumn{3}{|l|}{0.52} \\
\hline & Bio $(B)=$ & \multicolumn{3}{|l|}{$* *$} & \multicolumn{3}{|l|}{$* *$} \\
\hline & $\mathbf{S} \times \mathbf{C}=$ & \multicolumn{3}{|l|}{0.606} & \multicolumn{3}{|l|}{0.90} \\
\hline & $\mathbf{S} \times \mathbf{B}=$ & \multicolumn{3}{|l|}{0.529} & \multicolumn{3}{|l|}{0.75} \\
\hline & $\mathbf{C} \times \mathbf{B}=$ & \multicolumn{3}{|l|}{0.529} & \multicolumn{3}{|l|}{0.75} \\
\hline & $\mathrm{S} \times \mathrm{C} \times \mathrm{B}=$ & \multicolumn{3}{|l|}{0.916} & \multicolumn{3}{|l|}{1.30} \\
\hline
\end{tabular}


Journal of Environmental Sciences (JES)

Institute of Environmental Studies and Research, Ain Shams University

Mohamed, Asmaa et al.

Spraying plants with algae extract at $0.75 \mathrm{~cm} / 1$ gave a significant increase in all abovementioned yield components compared to the other two concentrations except for grains no. /spike which was the highest without spray of algae extract. Such results were the same in both seasons. It's clear that using algae as foliar biofertilizer showed a good means in that concern. These findings may be attributed to the role of algae as biofertilizer increasing total chlorophyll and enhancing the physiological activities in plants, which reflects on the activity of photosynthesis and plant growth characteristics. Results were corroborated with the findings of Mansour et. al., (2019) when has used algae at $1.5 \mathrm{~g} / \mathrm{l}$. Also, Attia and Abd El salam (2016) reported that, number of spike/m2 , number of grains per spike, 1000 grain weight were significantly increased with application of micro biofertilizer compared to control (without).

The application of compost with different rates included similar trend of algae extract. where the highest level of compost recorded the highest values of yield components except for grain no. /spike which was the highest without applied compost. Such results were similar in both seasons. The obtained results also showed that compost application induce promoting effect on yield and yield components of wheat. The enhancement effect of compost may be resulted from a greater concentration of plant nutrients like $\mathrm{N}, \mathrm{P}, \mathrm{K}$ and $\mathrm{Mg}$ and root rein for cement induced by compost (Donn et. al., 2014 and Nadjet et. al., 2014) .Similar results are obtained by Hafidi et al. (2012) who reported that biomass production of treated plants with $100 \mathrm{t} / \mathrm{ha}$ compost significantly 
Journal of Environmental Sciences (JES)

Institute of Environmental Studies and Research, Ain Shams University

Mohamed, Asmaa et al.

increased by $78 \%$ relative to the control.

The same tables showed that using biofertilizer took the same trend of the other two factors( algae extract and compost) in the two seasons. The treat ment of applied biofertilizer gave the highest significant values of yield components with exception of grain no./spike which was higher without applying biofertilizer.In this study, increases in agronomic criteria observed following inoculation with biofertilizer may be due to better utilization of nutrients in the soil through inoculation of efficient micro-organisms. A ppositive effect of biofertilizer on yield and yield components has been reported in the literature ( Migahed et. al., (2004) and Mansour et. al., (2019).

The interaction effect among the factors under study was accompanied with the individual effect of such factors. The highest level of studied factors $(0.75 \mathrm{~cm} / \mathrm{l}$ of algae extract, 8 ton compost/fad and application of biofertilizer $)$ resulted in the highest significant values of all yield components except that grains no./spike which was lower with such the abovementioned treatment ( no spray of algae extract, no addition of compost and biofertilizer). The previous results were the same in both seasons.

Wheat yield:The analysis of variance in Tables, $(8,9,10,11)$ showed the significant effect of the studied factors on grain, straw and biological yields ( $\mathrm{kg} / \mathrm{fad})$ In both seasons but harvest index \% was significantly affected by algae extract and compost in the $1^{\text {st }}$ season only and compost as well as the interaction between algae extract and compost in the $2^{\text {nd }}$ season. 
Table (8): Mean values of grain yield ( $\mathrm{kg} / \mathrm{fad})$ as affected by spray, compost and bio in 2016/2017 and 2017/2018 growing seasons

\begin{tabular}{|c|c|c|c|c|c|c|c|}
\hline \multirow{3}{*}{ Spray } & \multirow{3}{*}{ Compost } & \multicolumn{3}{|c|}{ Season $2016 / 2017$} & \multicolumn{3}{|c|}{ Season $2017 / 2018$} \\
\hline & & \multicolumn{6}{|c|}{ Mean grain yield } \\
\hline & & $\begin{array}{l}\text { Without } \\
\text { Bio }\end{array}$ & $\begin{array}{l}\text { With } \\
\text { Bio }\end{array}$ & $\begin{array}{l}\text { Total } \\
\text { Mean }\end{array}$ & $\begin{array}{l}\text { Witho } \\
\text { ut Bio }\end{array}$ & $\begin{array}{l}\text { With } \\
\text { Bio }\end{array}$ & $\begin{array}{l}\text { Total } \\
\text { Mean }\end{array}$ \\
\hline \multirow{3}{*}{$\begin{array}{l}\text { Witho } \\
\text { ut }\end{array}$} & Without & 1630.00 & 1843.33 & 1736.67 & 2032.00 & 2136.97 & 2084.48 \\
\hline & 6ton/fad & 2126.67 & 2251.67 & 2189.17 & 2413.60 & 2520.00 & 2466.80 \\
\hline & 8ton/fad & 2203.33 & 2323.33 & 2263.33 & 2476.93 & 2587.20 & 2532.07 \\
\hline \multicolumn{2}{|c|}{ Total Mean } & 1986.67 & 2139.44 & 2063.06 & 2307.51 & 2414.72 & 2361.12 \\
\hline \multirow{3}{*}{$\begin{array}{l}0.50 \mathrm{~cm} \\
/ 1\end{array}$} & Without & 1955.00 & 2383.33 & 2169.17 & 2245.60 & 2626.40 & 2436.00 \\
\hline & 6ton/fad & 2513.33 & 2725.00 & 2619.17 & 2716.00 & 2895.20 & 2805.60 \\
\hline & 8ton/fad & 2834.67 & 3276.00 & 3055.33 & 3001.60 & 3371.20 & 3186.40 \\
\hline \multicolumn{2}{|c|}{ Total Mean } & 2434.33 & 2794.78 & 2614.56 & 2654.40 & 2964.27 & 2809.33 \\
\hline \multirow{3}{*}{$\begin{array}{l}0.75 \mathrm{~cm} \\
/ 1\end{array}$} & Without & 2051.67 & 2446.67 & 2249.17 & 2340.80 & 2688.00 & 2514.40 \\
\hline & 6ton/fad & 2608.33 & 3060.00 & 2834.17 & 2805.60 & 3197.04 & 3001.32 \\
\hline & 8ton/fad & 2929.67 & 3413.33 & 3171.50 & 3080.00 & 3508.96 & 3294.48 \\
\hline \multicolumn{2}{|c|}{ Total Mean } & 2529.89 & 2973.33 & 2751.61 & 2742.13 & 3131.33 & 2936.73 \\
\hline \multicolumn{2}{|c|}{ Grand mean } & 2316.96 & 2635.85 & 2476.41 & 2568.01 & 2836.77 & 2702.39 \\
\hline \multirow{7}{*}{$\begin{array}{l}\text { LSD } \\
0.05\end{array}$} & Spray $(\mathbf{S})=$ & \multicolumn{3}{|l|}{32.059} & \multicolumn{3}{|l|}{46.08} \\
\hline & $\begin{array}{l}\text { Compost }(\mathrm{C} \\
=\end{array}$ & \multicolumn{3}{|l|}{45.164} & \multicolumn{3}{|l|}{23.01} \\
\hline & Bio $(\mathrm{B})=$ & \multicolumn{3}{|l|}{$* *$} & \multicolumn{3}{|l|}{$* *$} \\
\hline & $\mathrm{S} \times \mathrm{C}=$ & \multicolumn{3}{|l|}{78.226} & \multicolumn{3}{|l|}{39.86} \\
\hline & $\mathrm{S} \times \mathrm{B}=$ & \multicolumn{3}{|l|}{55.075} & \multicolumn{3}{|l|}{36.27} \\
\hline & $\mathrm{C} \times \mathrm{B}=$ & \multicolumn{3}{|l|}{ Ns } & \multicolumn{3}{|l|}{36.27} \\
\hline & $S \times C \times B$ & \multicolumn{3}{|l|}{95.392} & \multicolumn{3}{|l|}{62.82} \\
\hline
\end{tabular}


Journal of Environmental Sciences (JES)

Institute of Environmental Studies and Research, Ain Shams University

Mohamed, Asmaa et al.

Table (9): Mean values of biological yield ( $\mathrm{kg} / \mathrm{fad})$ as affected by spray, compost and bio in 2016/2017 and 2017/2018 growing seasons

\begin{tabular}{|c|c|c|c|c|c|c|c|}
\hline \multirow{3}{*}{ Spray } & \multirow{3}{*}{ Compost } & \multicolumn{3}{|c|}{ Season $2016 / 2017$} & \multicolumn{3}{|c|}{ Season 2017/2018 } \\
\hline & & \multicolumn{6}{|c|}{ Mean biological yield } \\
\hline & & $\begin{array}{l}\text { Witho } \\
\text { ut Bio } \\
\end{array}$ & $\begin{array}{l}\text { With } \\
\text { Bio }\end{array}$ & $\begin{array}{l}\text { Total } \\
\text { Mean } \\
\end{array}$ & $\begin{array}{l}\text { Witho } \\
\text { ut Bio }\end{array}$ & $\begin{array}{l}\text { With } \\
\text { Bio }\end{array}$ & $\begin{array}{l}\text { Total } \\
\text { Mean } \\
\end{array}$ \\
\hline \multirow{3}{*}{ Without } & Without & 3640.00 & 4086.67 & 3863.33 & 4451.00 & $\begin{array}{l}4679.3 \\
6 \\
\end{array}$ & 4565.18 \\
\hline & 6ton/fad & 4700.00 & 4977.67 & 4838.83 & 5280.80 & $\begin{array}{l}5516.0 \\
0 \\
\end{array}$ & 5398.40 \\
\hline & 8ton/fad & 4866.67 & 5130.00 & 4998.33 & 5416.93 & $\begin{array}{l}5658.8 \\
0 \\
\end{array}$ & $\mathbf{5 5 3 7 . 8 7}$ \\
\hline \multicolumn{2}{|c|}{ Total Mean } & 4402.22 & 4731.44 & 4566.83 & 5049.58 & $\begin{array}{l}5284.7 \\
2\end{array}$ & 5167.15 \\
\hline \multirow{3}{*}{$0.50 \mathrm{~cm} / \mathrm{l}$} & Without & 4320.00 & 5250.00 & 4785.00 & 4905.60 & $\begin{array}{l}5740.0 \\
0\end{array}$ & 5322.80 \\
\hline & 6ton/fad & 5536.67 & 5978.33 & $\mathbf{5 7 5 7 . 5 0}$ & 5947.20 & $\begin{array}{l}6333.6 \\
0\end{array}$ & 6140.40 \\
\hline & 8ton/fad & 6240.00 & 7177.33 & 6708.67 & 6580.00 & $\begin{array}{l}7380.8 \\
0 \\
\end{array}$ & 6980.40 \\
\hline \multicolumn{2}{|c|}{ Total Mean } & 5365.56 & 6135.22 & 5750.39 & 5810.93 & $\begin{array}{l}6484.8 \\
0\end{array}$ & 6147.87 \\
\hline \multirow{3}{*}{$0.75 \mathrm{~cm} / 1$} & Without & 4535.00 & 5398.33 & 4966.67 & 5118.50 & $\begin{array}{l}5882.2 \\
0 \\
\end{array}$ & 5500.35 \\
\hline & 6ton/fad & 5743.33 & 6673.33 & 6208.33 & 6143.20 & $\begin{array}{l}6999.4 \\
4\end{array}$ & 6571.32 \\
\hline & 8ton/fad & 6423.00 & 7495.00 & 6959.00 & 6748.00 & $\begin{array}{l}7719.7 \\
1\end{array}$ & 7233.86 \\
\hline \multicolumn{2}{|c|}{ Total Mean } & 5567.11 & 6522.22 & 6044.67 & 6003.23 & $\begin{array}{l}6867.1 \\
2\end{array}$ & 6435.18 \\
\hline \multicolumn{2}{|c|}{ Grand mean } & 5111.63 & 5796.30 & 5453.96 & 5621.25 & $\begin{array}{l}6212.2 \\
1\end{array}$ & 5916.73 \\
\hline \multirow{7}{*}{ LSD 0.05} & $\begin{array}{l}\text { Spray } \\
(\mathbf{S})= \\
\end{array}$ & \multicolumn{3}{|l|}{62.702} & \multicolumn{3}{|l|}{108.52} \\
\hline & $\begin{array}{l}\text { Compost } \\
(\mathrm{C}=\end{array}$ & \multicolumn{3}{|l|}{93.433} & \multicolumn{3}{|l|}{47.88} \\
\hline & Bio $(B)=$ & \multicolumn{3}{|l|}{$* *$} & \multicolumn{3}{|l|}{$* *$} \\
\hline & $\mathbf{S} \times \mathbf{C}=$ & \multicolumn{3}{|l|}{161.831} & \multicolumn{3}{|l|}{82.93} \\
\hline & $\mathbf{S} \times \mathbf{B}=$ & \multicolumn{3}{|l|}{115.591} & \multicolumn{3}{|l|}{79.56} \\
\hline & $\mathbf{C} \times \mathbf{B}=$ & \multicolumn{3}{|l|}{115.591} & \multicolumn{3}{|l|}{79.53} \\
\hline & $\begin{array}{l}S \times C \times B \\
=\end{array}$ & \multicolumn{3}{|l|}{200.210} & \multicolumn{3}{|l|}{137.80} \\
\hline
\end{tabular}


Table (10): Mean values of straw yield (kg/fad) as affected by spray, compost and bio in 2016/2017 and 2017/2018 growing seasons.

\begin{tabular}{|c|c|c|c|c|c|c|c|}
\hline \multirow{3}{*}{ Spray } & \multirow{3}{*}{ Compost } & Season 2 & $6 / 2017$ & & Season & $017 / 2018$ & \\
\hline & & \multicolumn{6}{|c|}{ Mean straw yield } \\
\hline & & $\begin{array}{l}\text { Without } \\
\text { Bio }\end{array}$ & $\begin{array}{l}\text { With } \\
\text { Bio }\end{array}$ & $\begin{array}{l}\text { Total } \\
\text { Mean }\end{array}$ & $\begin{array}{l}\text { Witho } \\
\text { ut Bio }\end{array}$ & $\begin{array}{l}\text { With } \\
\text { Bio }\end{array}$ & $\begin{array}{l}\text { Total } \\
\text { Mean }\end{array}$ \\
\hline \multirow{3}{*}{ Without } & Without & 2010.00 & 2243.33 & 2126.67 & 2419.00 & 2542.40 & 2480.70 \\
\hline & 6ton/fad & 2573.33 & 2726.00 & 2649.67 & 2867.20 & 2996.00 & 2931.60 \\
\hline & 8ton/fad & 2663.33 & 2806.67 & 2735.00 & 2940.00 & 3071.60 & 3005.80 \\
\hline \multicolumn{2}{|c|}{ Total Mean } & 2415.56 & 2592.00 & 2503.78 & 2742.07 & 2870.00 & 2806.03 \\
\hline \multirow{3}{*}{$0.50 \mathrm{~cm} / \mathrm{l}$} & Without & 2365.00 & 2866.67 & 2615.83 & 2660.00 & 3113.23 & 2886.62 \\
\hline & 6ton/fad & 3023.33 & 3253.33 & 3138.33 & 3231.20 & 3438.40 & 3334.80 \\
\hline & 8ton/fad & 3405.33 & 3901.33 & 3653.33 & 3578.40 & 4009.60 & 3794.00 \\
\hline \multicolumn{2}{|c|}{ Total Mean } & 2931.22 & 3340.44 & 3135.83 & 3156.53 & 3520.41 & 3338.47 \\
\hline \multirow{3}{*}{$0.75 \mathrm{~cm} / \mathrm{l}$} & Without & 2483.33 & 2951.67 & 2717.50 & 2777.60 & 3203.20 & 2990.40 \\
\hline & 6ton/fad & 3135.00 & 3613.33 & 3374.17 & 3337.60 & 3469.07 & 3403.33 \\
\hline & 8ton/fad & 3493.33 & 4081.67 & 3787.50 & 3668.00 & 4210.75 & 3939.38 \\
\hline \multicolumn{2}{|c|}{ Total Mean } & 3037.22 & 3548.89 & 3293.06 & 3261.07 & 3627.67 & 3444.37 \\
\hline \multicolumn{2}{|c|}{ Grand mean } & 2794.67 & 3160.44 & 2977.56 & 3053.22 & 3339.36 & 3196.29 \\
\hline \multirow{7}{*}{ LSD $_{0.05}$} & $\begin{array}{ll}\text { Spray } & (\mathbf{S}) \\
= & \end{array}$ & \multicolumn{3}{|l|}{32.470} & \multicolumn{3}{|l|}{163.76} \\
\hline & $\begin{array}{l}\text { Compost } \\
\text { (C = }\end{array}$ & \multicolumn{3}{|l|}{49.350} & \multicolumn{3}{|l|}{102.58} \\
\hline & Bio $(B)=$ & \multicolumn{3}{|l|}{$* *$} & \multicolumn{3}{|l|}{$* *$} \\
\hline & $\mathbf{S} \times \mathbf{C}=$ & \multicolumn{3}{|l|}{85.476} & \multicolumn{3}{|l|}{177.67} \\
\hline & $\mathbf{S} \times \mathbf{B}=$ & \multicolumn{3}{|l|}{61.622} & \multicolumn{3}{|l|}{143.68} \\
\hline & $\mathrm{C} \times \mathrm{B}=$ & \multicolumn{3}{|l|}{61.622} & \multicolumn{3}{|l|}{143.68} \\
\hline & $\begin{array}{l}\mathbf{S} \times \mathbf{C} \times \mathbf{B} \\
=\end{array}$ & \multicolumn{3}{|l|}{106.733} & \multicolumn{3}{|l|}{248.86} \\
\hline
\end{tabular}


Table (11): Mean values of harvest index $\%$ as affected by spray, compost and bio in 2016/2017 and 2017/2018 growing seasons.

\begin{tabular}{|c|c|c|c|c|c|c|c|}
\hline \multirow{3}{*}{ Spray } & \multirow{3}{*}{ Compost } & Season 2 & 6/2017 & & Season 20 & $/ 2018$ & \\
\hline & & \multicolumn{6}{|c|}{$\begin{array}{l}\text { Mean harvest index } \\
\end{array}$} \\
\hline & & $\begin{array}{l}\text { Without } \\
\text { Bio }\end{array}$ & $\begin{array}{l}\text { With } \\
\text { Bio }\end{array}$ & $\begin{array}{l}\text { Total } \\
\text { Mean }\end{array}$ & $\begin{array}{l}\text { Without } \\
\text { Bio }\end{array}$ & $\begin{array}{l}\text { With } \\
\text { Bio }\end{array}$ & $\begin{array}{l}\text { Total } \\
\text { Mean } \\
\end{array}$ \\
\hline \multirow{3}{*}{ Without } & Without & 44.78 & 45.09 & 44.93 & 45.66 & 45.66 & 45.66 \\
\hline & 6ton/fad & 45.23 & 44.89 & 45.06 & 45.70 & 45.68 & 45.69 \\
\hline & 8ton/fad & 45.26 & 45.28 & 45.27 & 45.72 & 45.72 & 45.72 \\
\hline \multicolumn{2}{|c|}{ Total Mean } & 45.09 & 45.08 & 45.09 & 45.69 & 45.69 & 45.69 \\
\hline \multirow{3}{*}{$0.50 \mathrm{~cm} / \mathrm{l}$} & Without & 30.79 & 33.52 & 32.15 & 45.78 & 45.75 & 45.76 \\
\hline & 6ton/fad & 35.75 & 37.49 & 36.62 & 45.66 & 45.70 & 45.68 \\
\hline & 8ton/fad & 38.44 & 43.76 & 41.10 & 45.61 & 45.67 & 45.64 \\
\hline \multicolumn{2}{|c|}{ Total Mean } & 45.34 & 45.53 & 45.44 & 45.68 & 45.71 & 45.70 \\
\hline \multirow{3}{*}{$0.75 \mathrm{~cm} / \mathrm{l}$} & Without & 45.21 & 45.32 & 45.27 & 45.72 & 45.69 & 45.71 \\
\hline & 6ton/fad & 45.41 & 45.84 & 45.63 & 45.67 & 45.67 & 45.67 \\
\hline & 8ton/fad & 45.59 & 45.53 & 45.56 & 45.64 & 45.45 & 45.55 \\
\hline \multicolumn{2}{|c|}{ Total Mean } & 45.40 & 45.56 & 45.48 & 45.68 & 45.61 & 45.64 \\
\hline \multicolumn{2}{|c|}{ Grand mean } & 45.28 & 45.39 & 45.34 & 45.68 & 45.67 & 45.68 \\
\hline \multirow{7}{*}{ LSD $_{0.05}$} & Spray $(\mathbf{S})=$ & \multicolumn{3}{|l|}{0.202} & \multicolumn{3}{|l|}{ Ns } \\
\hline & $\begin{array}{l}\text { Compost }(\mathrm{C} \\
=\end{array}$ & \multicolumn{3}{|l|}{0.122} & \multicolumn{3}{|l|}{0.06} \\
\hline & $\operatorname{Bio}(\mathbf{B})=$ & \multicolumn{3}{|l|}{ Ns } & \multicolumn{3}{|l|}{ Ns } \\
\hline & $\mathrm{S} \times \mathrm{C}=$ & \multicolumn{3}{|l|}{ Ns } & \multicolumn{3}{|l|}{0.10} \\
\hline & $\mathbf{S} \times \mathbf{B}=$ & \multicolumn{3}{|l|}{$\frac{\mathrm{Ns}}{\mathrm{Ns}}$} & \multicolumn{3}{|l|}{ Ns } \\
\hline & $\mathbf{C} \times \mathbf{B}=$ & \multirow{2}{*}{\multicolumn{3}{|c|}{$\frac{\mathrm{Ns}}{\mathrm{Ns}}$}} & \multirow{2}{*}{\multicolumn{3}{|c|}{$\frac{\mathrm{Ns}}{\mathrm{Ns}}$}} \\
\hline & $\mathrm{S} \times \mathrm{C} \times \mathrm{B}=$ & & & & Ns & & \\
\hline
\end{tabular}


Journal of Environmental Sciences (JES)

Institute of Environmental Studies and Research, Ain Shams University

Mohamed, Asmaa et al.

Spraying wheat plants with algae extract at concentration of $0.75 \mathrm{~cm} / 1$ achieved marked superiority and gave the highest values of grain, straw and biological yields in both seasons compared to the other two concentrations. While the highest values of harvest index\% were obtained with concentration $(0.75 \mathrm{~cm} / 1)$ or $(0.50 \mathrm{~cm} / 1)$ with no significant difference between them in the $1^{\text {st }}$ season only These results confirm the efficiency of the algae extract as biofertilizer that regulate the balance between

photosynthesis and respiration processes in plants. Similar trend was obtained by Rasha et al (2020) who showed that there was a significant effect of algae extract foliar spraying on grain straw and biological yields in both seasons.

Different levels of compost treatments had a significant influence on wheat yields. The application of the highest level of compost ( 8 ton/fad.) recorded significantly the highest increase for grain, straw and biological yields as well as harvest index $\%$ in both seasons comparing with the other compost rates. Multi benefits' derived from compost using as fertilizer by increasing organic content and microbial activity (Scotti et al 2015). A great concentration of plant nutrients like $\mathrm{N}, \mathrm{P}, \mathrm{K}$ and $\mathrm{Mg}$ resulted from compost application (Donn et al 2014).

The application of biofertilizer significantly increased wheat yield i.e. grain, straw and biological ones but harvest index \% didn't significantly affected by such factor. These results were the same in both seasons. These results are in agreement with those of Mansour et al (2019) and Migahed et all (2004). 
Journal of Environmental Sciences (JES)

Institute of Environmental Studies and Research, Ain Shams University

Mohamed, Asmaa et al.

With respect to the interaction effect of the studied factors, data showed that wheat yields significantly affected by the interacted factors under study. It can be mentioned that wheat plants sprayed with algae extract at $0.75 \mathrm{~cm} / 1$ concentration and fertilized with 8 ton/fad compost and biofertilizer were significantly superior compared to the other treatments in the $1^{\text {st }}$ season but harvest index \% didn't significantly affect by such interaction except of the interaction between algae extract and compost where spraying wheat plants with algae extract at $0.50 \mathrm{~cm} / 1$ without compost application gave the highest significant value of harvest index $\%$ in the $2^{\text {nd }}$ season.

\section{CONCLUSION}

From the obtained results in this study, it can be concluded that planting wheat plants sprayed with $0.75 \mathrm{~cm} / 1$ of algae extract with compost and biofertilizer were maximized wheat productivity at Mashtool El -Souk, AlSharkia Governorate, Egypt.

\section{REFERENCES}

A.O.A.C, (1970). Official Methods of Analysis if association of Official Agricultural Chemists 11th ed. Published by the A.O.A.C., P. O. Box. 540, Washington, D. C.

Attia , M.A and Abd El salam, A.A. (2016) . effect of mineral, organic and bio- fertilizer on yield and yield components of bread wheat at Siwa Oasis. Alex. J. Agric. Sci., 61(3):211-219. 
Awad, E.M.; Youssef , N.S. and Shall, Z.S. (2006). Effect of foliar spraying seaweed extracts and inorganic fertilizer levels on growth, yield and quality of potato crop. J. Agric. Sci., Mansoura Univ., 31(10): 6549-6559.

Black, C. A. (1982). Methods of Soil Analysis. Part 2. Chemical and Microbiological Properties. $2^{\text {nd }}$ edition. Amer. Soc. of Agron. Madison, Wisconsin, USA.

Donn, S.; Wheatly, R.E.; Mckenzie B.M.; Loades, K.W.; Hallett PD(2014). Improved soil fertility from compost amendment increases root growth and reinforcement of surface soil on slop. Ecol Eng 71:458-465.

El Habbasha, S.F.; Hozayn, M. \& Khalafalla, M.A. (2007). Integration effect between phosphorus levels and bio-fertilizers on quality and quantity yield of faba bean (Vicia faba L.) in newly cultivated sandy soils research. J. Agric. \& Biol. Sci., 3(6). 966-971.

FAO (2020). Food and Agriculture Organization of the United Nations, FAOSTAT, FAO Statistics Division 2019, March 2020.

Hafidi M, Amir S, Meddich A, Jourrrraibhy A, Wintttterton P, El Gharous M, Duponnois R (2012). Impact of applying composted biosolids on wheat growth and yield parametrers on a calcimagnesic soil in a semi-arid region Afr J biotechnol 11(41):9805-9815.

Hall, J.L. and Williams, L. E. (2003): Transition metal transporters in plants. J. of Experimental Botany, 54(393): 2601-2613.

Imran, M. and Gurmani, Z.A. (2012): Role of macro and micronutrients in the plant growth and development. Sci. Tech. and Devel., 30(3): 3640 .

Jackson, M.L. (1973): Soil Chemical Analysis. Prentice hall, Englewood chiffs-N Jeresey, USA. Hall of India, New Delhi. 
Karthikeyan, K. and Shanmugam, M. (2015):Yield and oil content of peanut(var. TMV-7) and sunflower (var. co-2) applied with bio stimulant AQUASAP manufactured from seaweed. African J, of Agric. Res., 10(10):1031-1042.

Latique, S.; Chernane, H.; Mansori, M. and El Kaoua, M. (2013). Seaweed liquid fertilizer effect on physiological and biochemical parameters of bean plant (Phaseolus vulgaris variety paulista) under hydropnic system. Euro. Scient. J., 9(30): 174-191.

Mansour, H.A.; Nofal,O.A. Gaballah, M.S. and El- Nasharty, A.B. (2019). Impact of algae extract foliar application on two wheat varieties with using two irrigation systems . Bioscience Res., 16(1):356366.

Migahed H A, Ahmed AE, Abd EL-Ghany BF(2004). Effect of different bacterial strains as biofertilizer agents on growth, production and oil of Apium gaveolense under calcareous soil.J. Agric. Sci. 12:511-525.

Nadjet M, Abderezzak D, Meeeriem KH (2014). Effect of three types of composts of olive oil by- products on growth and yield of hard wheat "Triticcum durum Desf". Afr J Biotechnol 13(52):46854693.

Nofal, O.A.; Hellal, F.A.; El-Sayed, S.A.A. and Bakry, A.B. (2016). Response of peanut and maize crops to foliar application of algae extracts under sandy soil condition. Res. J. of Pharmace. , Biolo. and Chem. Sci.,7(5):15-157. 1

Pramanick, B.; Brahmachari ,K. and Ghosh, A. (2013). Effect of seaweed saps on growth and yield improvement of green gram. African J. Agric. Res., 8(13): 1180-1186.

Prasad, K.; Das, A.K.; Siddhanta,A.K.; Oza, M.D.; Brahmbhatt,H. Meena,R. Eswaran, K. Rajyaguru, M.R. and Ghosh, P.K. ( 2010): Detection and quantification of some plant growth regulators in a seaweedbased foliar spray employing a mass spectrometric technique sans 
chromatographic separation. J. Agric. Food Chem., 58(8): 45944601 .

Reddy, S.R.(2004): Agronomy of Field Crops. Ludhiana, Kalyani Publishers. ISBN: 9788127248994-8127248991, p.143.

Ryan, J.; Garabet, S.; Harmsen, K. and Rashid, A. (1996): A Soil and Plant Analysis. Manual Adapted for the west Asia and North Africa Region. ECARDA, Aleppo, Syria. 140 pp.

Rasha S.A.ELMoursy; A.A.A. Leilah; Soad H. Haffez and M.A. Badwi (2020). Response of wheat to mineral Nitrogen levels and foliar Application with Algae extract. J.of plant production.; 11(4):349354.

Scotti, R.; Ascoli, D.R.; Bonnanomi, G.; Caceres, M.G.; Sultana, S.; Cozzolino, L.; Scelza, R.; Zoina, A.; Rao, M.A. (2015). Combined use of compost and wood scraps to increase carpon stock and improve soil quality in intensive farming systems. Eur.J Soil Sci.http://doi. Org/10.1111/ejss.12248.

Shaaban, M.M.; El-Saady, A.M. and El-Sayed, A.B. (2010).Green microalgae water extract and micronutrients foliar application as promoters to nutrient balance and growth of wheat plants. J. of American Sci., 6(9): 631-636.

Shalaby, T.A. and H. El-Ramady (2014). Effect of foliar application of biostimulants on growth, yield, yield components, and storability of garlic (Allium sativum L.). AJCS., 8(2): 271-275.

Snedecor, G. W. \& Cochran, W. G. (1980). One way classification Analysis of Variance - The random effect model - Two way classification (Eds) In Statistical Methods. The Iowas state Univ., Press Ames Iowa, USA: 215-273.

Waller, R. A. \& Duncan, C. B. (1969). A basyan rule for symmetric multiple comparison problem. Amer. State Assoc. J. December: 14851503. 
Zhang, X. and E.H. Ervin (2004). Cytokinin-containing seaweed and humic acid extracts associated with creeping bentgrass leaf cytokinins and drought resistance. Crop Sci., 44(5): 1737-1745.

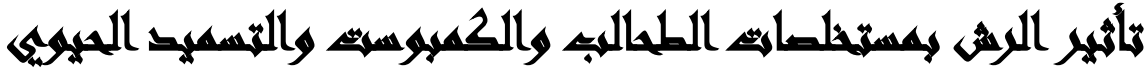

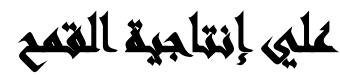

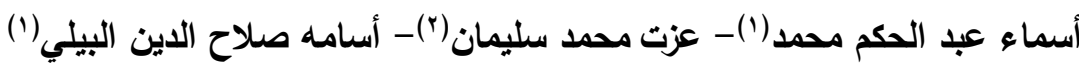

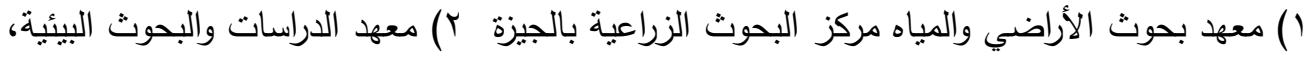
جامعة عين شمس بـ الارض

\section{المستخلصى}

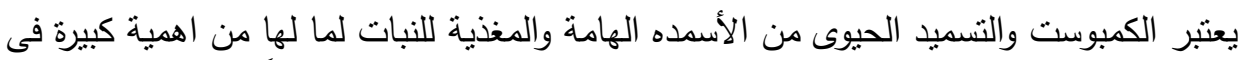

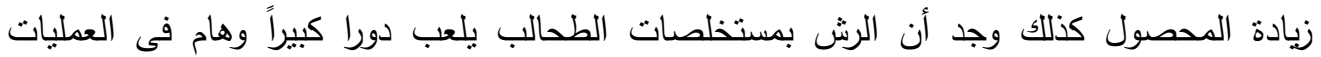

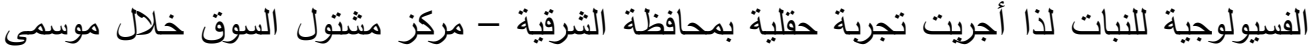

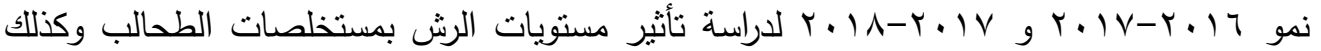

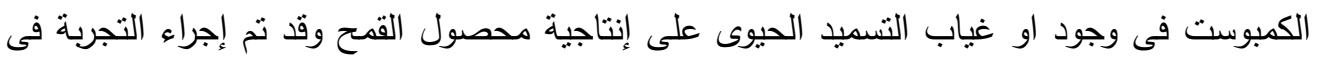

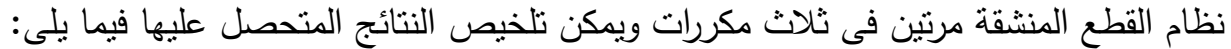

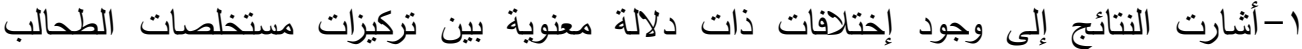

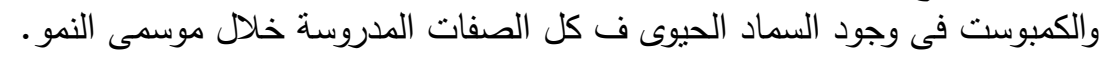

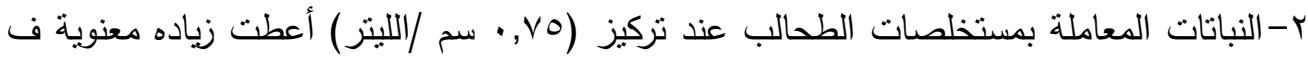

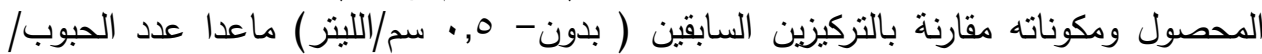

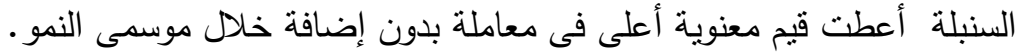

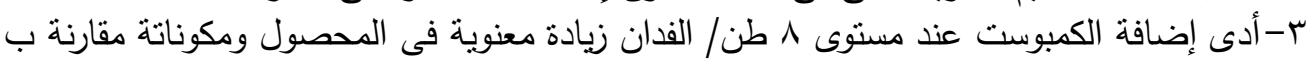

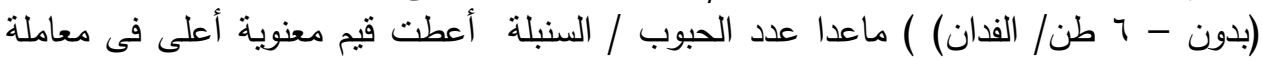
الكنترول خلال الموسمين. ؟--إضافه التسميد الحيوى اخذ نفس إتجاه الرش بمستخلصات الطحالب والكمبوست فى موسمى 
Journal of Environmental Sciences (JES)

Institute of Environmental Studies and Research, Ain Shams University

Mohamed, Asmaa et al.

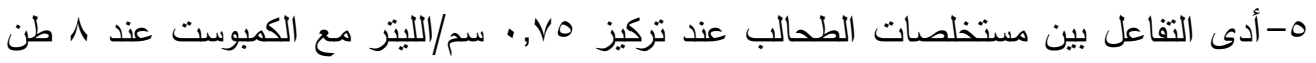

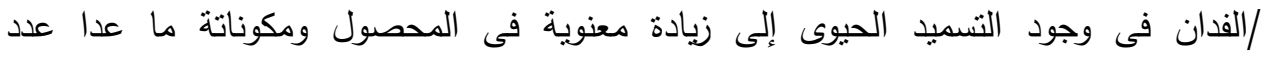

الحبوب/السنبلة فى الموسمين. 